This item was submitted to Loughborough's Research Repository by the author.

Items in Figshare are protected by copyright, with all rights reserved, unless otherwise indicated.

\title{
A framework for predicting the non-visual effects of daylight - part I: photobiology-based model
}

PLEASE CITE THE PUBLISHED VERSION

http://dx.doi.org/10.1177/1477153511435961

\section{PUBLISHER}

Sage, published in Association with The Society of Light and Lighting (@ The Chartered Institution of Building Services Engineers)

\section{VERSION}

AM (Accepted Manuscript)

\section{LICENCE}

CC BY-NC-ND 4.0

\section{REPOSITORY RECORD}

Andersen, Marilyne, John Mardaljevic, and S.W. Lockley. 2019. "A Framework for Predicting the Non-visual Effects of Daylight - Part I: Photobiology-based Model”. figshare. https://hdl.handle.net/2134/11567. 
This item was submitted to Loughborough's Institutional Repository (https://dspace.lboro.ac.uk/) by the author and is made available under the following Creative Commons Licence conditions.

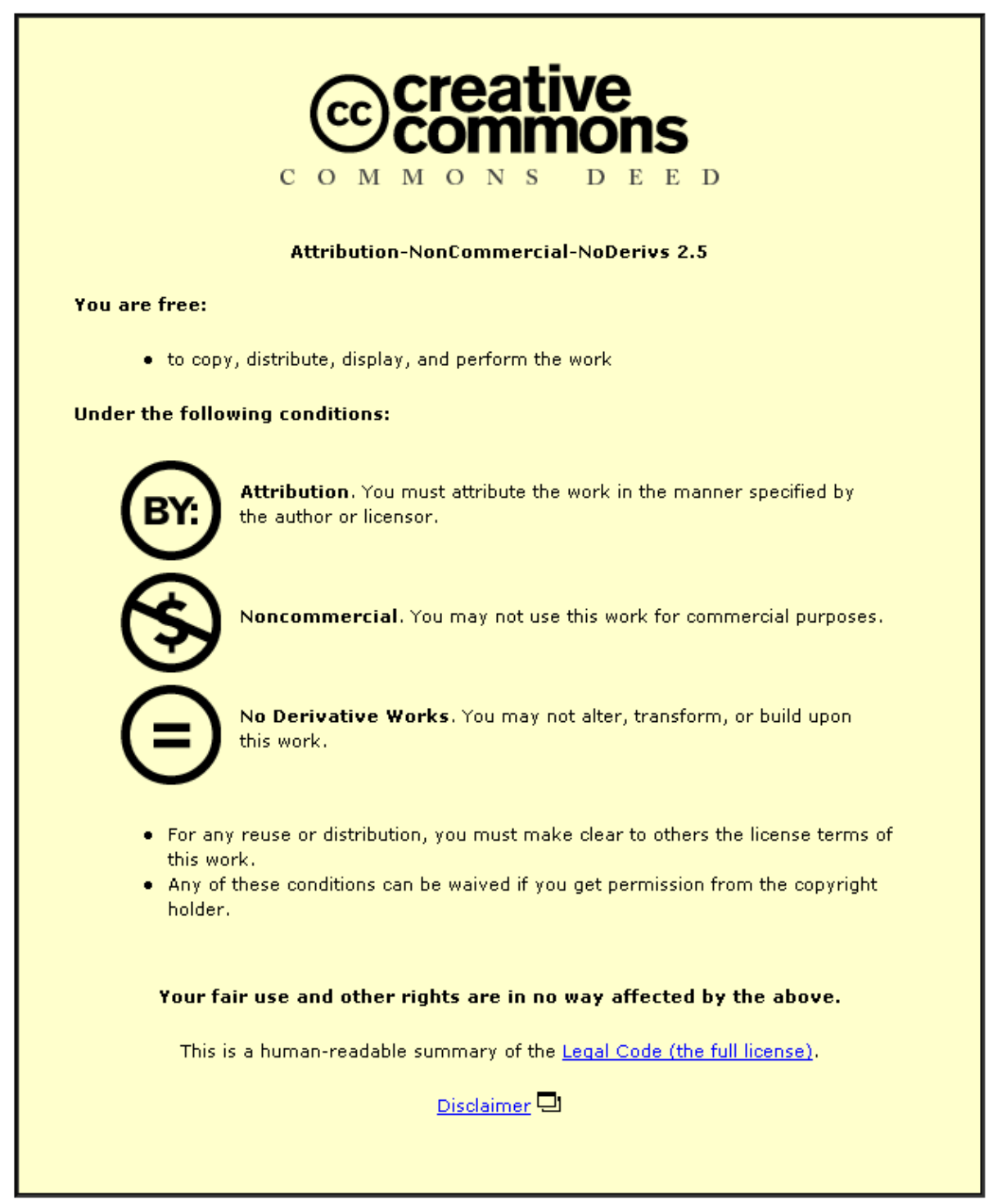

For the full text of this licence, please go to: http://creativecommons.org/licenses/by-nc-nd/2.5/ 


\section{A Framework for Predicting the Non-Visual Effects of Daylight - Part I: Photobiology-based Model}

Marilyne Andersen ${ }^{1, *}, \mathrm{PhD}$, John Mardaljevic ${ }^{2}, \mathrm{PhD}$, and Steven W. Lockley, $\mathrm{PhD}^{3}$,

${ }^{1}$ Interdisciplinary Laboratory of Performance-Integrated Design (LIPID), School of Architecture, Civil and Environmental Engineering (ENAC), Ecole Polytechnique Fédérale de Lausanne (EPFL), Lausanne, Switzerland

${ }^{2}$ Institute of Energy and Sustainable Development (IESD), De Montfort University, Leicester, UK

${ }^{3}$ Division of Sleep Medicine, Department of Medicine, Brigham and Women's Hospital and Division of Sleep Medicine, Harvard Medical School

\section{short title: Non-Visual Effects of Daylight I}

* Corresponding author. Address for correspondence: Prof. M. Andersen, EPFL-ENAC-IA-LIPID, BP 2229 (Building BP), Station 16, 1015 Lausanne, Switzerland. Phone: +41 2169308 82, Email: marilyne.andersen@epfl.ch

Word count: 6765 


\begin{abstract}
This paper (part one of two) investigates the formulation of metrics for the nonvisual effects of daylight, such as entrainment of the circadian system and a maintenance of alertness. The body of empirical data from photobiology studies is now sufficient to allow us to develop preliminary non-visual lighting evaluation methods for lighting design. These effects have the potential to become a relevant quantity to consider when assessing the overall daylighting performance of a space. This paper (Part I) describes the assumptions and general approach that were developed to model occupant exposure to nonvisual effects of light, and presents a novel means of visualizing the 'circadian potential' of a point in space. The proposed approach uses outcomes of photobiology research to define threshold values for illumination in terms of spectrum, intensity, and timing of light at the human eye. These values are then translated into goals for lighting simulation, based on vertical illuminance at the eye, that - ultimately - could become goals for building design. A new climatebased simulation model was developed to apply these concepts to a residential environment, described in Part II (forthcoming).
\end{abstract}




\section{Introduction}

The primary concern in the daylighting of buildings has generally been to provide illumination for task, for example to ensure that 500 lux falls on the horizontal work plane. Although climate-based daylight modeling is over a decade old ${ }^{1,2}$, building guidelines and recommendations worldwide are still founded on idealized, static sky conditions such as the CIE standard overcast sky (i.e. to predict daylight factors). It is only recently that daylight metrics founded on climate-based simulations have begun to be considered as the basis for the next generation of building guidelines ${ }^{3}$, thus enabling a more realistic and location-specific evaluation of daylighting potential. In the last decade, there has also been a gradual increase in awareness of the non-visual effects of daylight (or more generally light) received by the eye ${ }^{4}$. It is well-known that building occupants almost without exception will prefer a workstation with a view of the outdoor environment to a windowless office ${ }^{5}$. A view to the outside of course indicates the presence of daylight, although the relation between view and daylight provision is not straightforward as it is dependent on many factors. In addition to subjective preferences for daylit spaces, it is now also firmly established that light has measurable neuroendocrine and neurobehavioral effects on the human body, in particular with respect to ensuring a healthy sleep - wake cycle and maintaining alertness. Could the quality and nature of the internal daylit environment have a significant effect on human health? Evidence is indeed suggestive of links between daylight exposure and both health and productivity ${ }^{6}$.

The daily cycle of day and night plays a major role in regulating and maintaining 24-hour rhythms in many aspects of our physiology, metabolism 
and behaviour.. These daily rhythms can be termed circadian rhythms - the term "circadian" is derived from the Latin circa, "around", and diem or dies, “day", meaning literally "approximately one day" - and the circadian system is commonly referred to as the 'body clock'. Circadian rhythms occur in almost all organisms from bacteria to mammals and are endogenous, meaning that they are spontaneously generated from within the organism even in the absence of external or environmental time cues, These internal rhythms do not usually have a period of exactly 24 hours and therefore the cycles need to be adjusted or entrained to environment time by external cues, the primary one of which is the daily light-dark cycle.

The primary circadian "clock" in mammals is located in the suprachiasmatic nuclei (SCN), a pair of hypothalamic nuclei containing about 50,000 cells. The $\mathrm{SCN}$ receives light information exclusively via the eyes. The retina of the eye contains not only the well-known photoreceptors which are used to detect light for vision (i.e. rod and cones) but also contain a subset of specialized retinal ganglion cells that are intrinsically photosensitive and project directly to the $\mathrm{SCN}$ and other brain areas mediating 'non-visual' responses to light. The SCN also conveys signals to other parts of the brain to control the timing of numerous circadian rhythms, including the sleep-wake cycle, performance and alertness patterns, hormones such as melatonin and cortisol, and the core body temperature rhythm. Light also has several acute effects in addition to synchronizing the circadian clock. First, pineal melatonin, which is considered the biochemical correlate of darkness, is acutely suppressed by light exposure at night via the same retina-SCN-pineal pathway that generates melatonin and synchronizes its rhythms ${ }^{7,8}$. Second, light is a stimulant and has direct alerting 
effects in the brain including measurable effects on subjective sleepiness ratings, reaction time and cognitive performance, and brain activity ${ }^{9}$.

The failure to maintain normal entrainment of the circadian system to the natural 24-hour cycle of daylight results in many negative health outcomes for humans, though not all are fully understood. Jet Lag and Shift Work Disorders are examples of clinical Circadian Rhythm Sleep Disorders in which the internal circadian clock is not synchronized to the external light-dark cycle. For example, when traveling across a number of time zones, the circadian system cannot immediately reset to the new light-dark cycle (it takes about a day per time zone to adjust) and consequently will be 'desynchronized' from environmental time ${ }^{9}$. Similarly, shiftworkers induce a circadian disorder by choosing to work during the night and sleep during the day, in opposition of the natural circadian cycle ${ }^{9-11}$, inducing both short-term risks for sleepiness related accidents and injuries, and longer-term risks to health ${ }^{12-15}$ and worker productivity $^{16-17}$.

The timing, intensity, spectrum, duration, pattern of light received at the eye, and prior light history, are the principal factors determining entrainment of the circadian cycle6. Inadequate or mistimed light exposure can disrupt normal circadian rhythms and have a negative effect on human performance, alertness, health or safety. The type and timing of light exposure is often influenced by the built environment, especially in dense urban settings. Daylight usually provides illuminances significantly higher than the design level required for visual function, though this is only in close proximity to windows and perhaps also highly daylit spaces such as atria. If the typical illuminances in these zones are high - but not so great that blinds are needed - then those building users 
that regularly occupy the well-daylit spaces may perhaps experience stronger and more regular 24-hour light dark cycles, and consequently more robust circadian entrainment, than those users positioned away from windows who are habitually exposed to lower illuminance levels at the eye. These considerations have resulted in the notion that a space - through its interaction with daylighting - may possess a certain 'circadian potential" ${ }^{18}$.

Given the emergent nature of these concepts, it should be understood that the process of determining this 'circadian potential' is more one of carefully considered judgment than an as-yet commonly agreed procedure: the right balance between optimizing light simultaneously for both visual and non-visual functions has not yet been found, nor do we know how to model the light responses accurately, especially during the day. The vast majority of circadian photoreception studies - mostly using monochromatic light or stationary electric lighting - have indeed been conducted at night or in dim-light conditions and been focusing on non-visual effects at the onset of light exposure $^{19-21}$; only small pilot studies have started to address longer-term daytime exposure ${ }^{22,23}$. Notwithstanding this caveat, a workable schema was devised by Pechacek and ourselves to begin applying this process specifically to lighting design ${ }^{18}$, that included a static 'circadian efficacy' concept similar to other recently proposed circadian action functions ${ }^{24-27}$.

As the state of the art in terms of daytime circadian studies is still embryonic, we are aiming for a modeling framework - rather than a metric - that will evolve as photobiology findings get more refined and applicable. While strict recommendations cannot yet be made, the relevance of some critical design parameters on the perceived light spectrum, intensity, duration and other 
parameters is certainly a topic of investigation: we know that we have dual light detection systems, whose components have differing spectral sensitivities and whose combined effect will vary depending on timing, intensity, wavelength, pattern and exposure duration factors. Without yet trying to propose design guidelines, we want to move towards lighting models that allow interdependencies of these different parameters to be revealed and that offer enough flexibility to incorporate new developments in photobiology.

The goal of this paper is to make a first step in that direction - to begin the development of calculation methods and simulation workflows that allow us to extract circadian-relevant information from traditional, vision-based building simulation results, and to propose data analysis and visualization procedures that can meaningfully present and interpret these results. With these in place, the daylight exposure and timing influenced by design and environmental factors such as opening size and orientation, climate type, or dominant view directions can then be evaluated prospectively.

\section{Relevant findings from the photobiology field}

Two action spectra were determined for the melatonin suppression response in humans as the first comprehensive efforts to determine the spectral sensitivity of 'non-visual' responses to light in humans ${ }^{28,29}$. These action spectra exhibited a peak sensitivity in the visible short-wavelength (blue) light range (446$477 \mathrm{~nm}$ ) that was consistent with the discovery of novel blue-light sensitive photopigment called melanopsin located in the specialized intrinsically

photosensitive retinal ganglion cells ${ }^{30}$. The sensitivity curve that peaks in the 
blue region of the spectrum is illustrated in Figure 1: the one shown - for now called $\mathrm{C}(\lambda)$ - is based on that derived by Pechacek, Andersen \& Lockley ${ }^{18}$ and is represented alongside the well-known human photopic curve $\mathrm{V}(\lambda)$ that has a peak value at $555 \mathrm{~nm}$.

Figure 1: Spectral responses of visual system (photopic curve $V(\lambda)$ ) and circadian system (melanopsin action spectrum $C(\lambda)$ ).

This analysis used the dose-response curve determined by Cajochen et al in 2000 for the direct alerting effects of light during a 6.5-hour night-time exposure to $4100 \mathrm{~K}$ polychromatic light ${ }^{31}$. That study found that a corneal illuminance of about 300 lux - based on a visual reading of the plotted doseresponse curve - was required to achieve a maximal stimulant effect on subjective alertness under these conditions.

As mentioned earlier, very few alertness studies for daytime exposure to polychromatic light are available ${ }^{32}$, and none provides a dose-response curve. One daytime study of note was reported by Phipps-Nelson et al in $2003^{33}$ : it compared the effect on alertness of daytime exposure to bright (1000 lux) and $\operatorname{dim}(<5$ lux) light for 5 hours. The latter was assessed through measures of subjective and objective sleepiness for slightly sleep-deprived subjects, and again - used fluorescent lighting. Unlike previous related studies ${ }^{34,35}$ that used higher 'dim' light levels (50 lux e.g.), this one reported a significant effect of bright light exposure during daytime, probably due to the combination of having particularly dim comparison levels and having sleep-deprived subjects. 
Lastly, in relation to timing, early evening and nightime light exposure has been found to result in delaying the circadian clock i.e. in extending our biological day, while early to mid-morning light will advance the clock (shorten our biological day). The direction and magnitude of circadian phase resetting effects with respect to time of light exposure are described by a Phase Response Curve, one example of which is found in Khalsa et $\mathrm{al}^{9,36}$. While individual phase responses to light will depend on individual differences in circadian phase, a nominal definition has been used here which assumes that the core body temperature minimum $\left(\mathrm{CBT}_{\min }\right.$ ) time (an approximation of the point at which light switches from causing phase delays to phase advances) is at $6: 00 \mathrm{~h}$, for an individual typically sleeping from $0:: 00-8: 00 \mathrm{~h}$. Using this definition, light exposure before $\mathrm{CBT}_{\text {min }}(\sim 18: 00-6: 00 \mathrm{~h})$ will induce a phase delay of the clock where light exposure after $\mathrm{CBT}_{\min }(6: 00-18: 00 \mathrm{~h})$ will cause an advance. The effect is not equal at all times, however; the PRC predicts peak delay shifts around around $3 \mathrm{am}$ and peak advances around $9 \mathrm{am}$. In terms of alerting effects, the impact of light exposure or avoidance will also differ with timing. For example, while morning light exposure might be effective in accelerating the reduction in sleep inertia, or the 'grogginess' when one wakes up ${ }^{37}$, evening exposure to light could lead to detrimental effects on subsequent sleep latency and quality because of an undesired increase in alertness too late in the day ${ }^{38}$.

One should note that the history and duration of light exposure also have a critical influence on how the circadian system is stimulated ${ }^{39-41}$. Any light exposure threshold will thus be strongly influenced by these parameters, and with inter-dependencies that have not yet been fully uncovered. Research in this area is, as of yet, not advanced enough to provide more tangible hypotheses 
about how these dependencies should be applied in a daylight exposure context, and thus were considered beyond the scope of the model presented here.

\section{Incorporating non-visual effects in lighting simulation models}

The above-mentioned action spectrum, illuminance thresholds and time cues were used as a basis for setting up a non-visual lighting model with the aim to provide insights as to if and when one might expect a non-visual effect (e.g. alertness increase) during either nighttime or daytime in a daylit space, and thus allow a prospective assessment of the 'circadian potential' of a space, which will emerge from a complex map of inter-relationships at the human, spatial and environmental levels conceptually illustrated in Figure 2.

Figure 2: Uncertainties related to context variability and space properties integrated in a relationship map between measurable (scientific) parameters and design elements.

\subsection{Spectral properties and conversion factors}

To determine light levels relevant to our circadian photoreception system (as opposed to our visual system $)^{42}$, what we need to do is to convert predictions for vertical illuminance - derived from our visual system's sensitivity curve $\mathrm{V}(\lambda)$ - into equivalent 'circadian-lux' based on the $\mathrm{C}(\lambda)$ action spectrum given in Figure 1. 
Given the significant spectral shift from the $V(\lambda)$ to the $C(\lambda)$ curve, we can only get to comparable 'circadian-lux' values if we first identify the spectral distribution of the light reaching the eye, and then keep a consistent basis for 'circadian-lux' comparisons. In this case, only daylight will be considered as the light source although the method is conceptually equally applicable to electric lighting as long as the respective luminaire emission spectra are known.

The climate-based simulation method that was developed for this project is able to produce time-series of illuminance values independently for overcast sky light, sunlight and clear (blue) sky light (described in Part II). The sky model type (e.g. overcast, intermediate, clear) is inferred from diffuse horizontal and direct normal illuminance in the climate data ${ }^{43}$. Vertical daylight illuminance at the eye for a person inside a building can thus be considered as a set of individual contributions from direct and reflected sunlight, and diffuse daylight from either a clear blue sky or a grey overcast sky. Each partial illuminance value can then be converted into an equivalent partial 'circadian illuminance' using the approach described in Pechacek, Andersen \& Lockley ${ }^{18}$.

The respective spectral distributions actually applicable for these three daylight components at any given moment over the year will vary with the associated sky conditions specific to the considered situation. As the purpose of this study is not to provide a precise analysis of daylight penetration in a space but rather to define a general methodology for a non-visual lighting model applicable to existing building simulation workflows, we will approximate each of these three daylight components to a CIE standard illuminant: solar beam radiation will be approximated to D55, overcast sky to D65 and light from a clear blue sky to D75; all three are given in Figure 3. 
The conversion then comes down to determining the 'circadian efficacy' of the daylight component, starting from a known (visual) illuminance and relative spectral distribution from which an absolute radiometric spectrum can be derived (that will be different for each daylight component). The mathematical process used to calculate this 'circadian efficacy' can be found in Pechacek, Andersen \& Lockley $^{18}$ and can be summarized as follows: by multiplying the absolute spectrum by the 'circadian' sensitivity curve $C(\lambda)$ discussed above, one can then extract a 'circadian-lux' value for each component. The normalization factor of $683 \mathrm{~lm} / \mathrm{W}$ used for photometry is here replaced with an arbitrary value of 1 given that we are looking at determining a unit-less photometric response from a unit-less radiometric spectrum.

Through this conversion, one can account for the greater 'circadian efficacy' of, say, 1000 lux of diffuse light from a clear blue sky compared to 1000 lux of light from the sun. We can then use these established relationships to derive 'circadian-equivalent' threshold values derived from the photobiology findings discussed in section 2, as detailed below in section 3.2.

Figure 3: Spectral power distribution for CIE daylight illuminants associated to the three daylight sources alongside normalized photopic and circadian sensitivity curves $V(\lambda)$ and $C(\lambda)$.

Of course, the spectrum of light that ultimately reaches the eye will also be altered when it is transmitted through glass and when it is reflected off internal and external surfaces before reaching the eye. As a first approximation, we will consider that all surfaces and glazings that daylight will encounter (e.g. window glazing, walls, floor, ceiling, ground, etc.) to be spectrally neutral (achromatic 
i.e. 'grey'), and so do not modify the relative spectrum of light by reflection or transmission (though of course, they do modify the absolute values through absorption). This simplifying assumption will need to be tested, though for

spaces with clear glazing and neutral décor, a preliminary sensitivity analysis ${ }^{18}$ indicated that the effects were usually small except for very deep rooms. Thus, the calculated vertical illuminance only has to be split into the different daylight sources involved - which have a distinct relative spectra - following the procedure explained above.

\subsection{Intensity of illumination}

Based on the $C(\lambda)$ efficacy curve and the method described above, it becomes possible to determine the (visual) illuminance that, for a given source, would correspond to the same 'circadian-equivalent' illuminance for another source. For example, 190 lux of Daylight Illuminant D65 would correspond to 700 lux of $555 \mathrm{~nm}$ monochromatic light in terms of circadian effectiveness. Figure 4 shows this correspondence for a range of well-known illuminants and source types and proposes a correlation to subjective alertness based on the night-time study by Cajochen et. al. ${ }^{31}$. From that reference study, which provides illuminance threshold values for $25 \%, 50 \%, 75 \%$ and $100 \%$ subjective alertness for a single light source (4100 $\mathrm{K}$ fluorescent lighting), one can derive prospective thresholds for other sources, for which some examples are shown in Figure 4.

Figure 4: Illuminance necessary to achieve a desired relative alerting effect for a selection of wellknown illuminants . 
While it is too early to derive any reliable illuminance threshold for alertness from the night-time and daytime studies published so far, we can anticipate that daytime 'circadian-lux' thresholds are likely to be determined in the future in association with subjective and objective alertness, as well as other physiological and health effects. Until these more reliable thresholds are determined, and the duration of exposure is more reliably characterized, we can prospectively use the dose response curve from the night-time Cajochen study ${ }^{31}$ in combination with the daytime Phipps-Nelson results ${ }^{33}$ as workable basis for the development of a non-visual lighting model that will be further refined in the future as photobiology research progresses. Consequently, what we propose here is to use the Cajochen and Phipps-Nelson studies to, respectively, set a tentative lower and and an upper bound for the likelihood that a given light exposure will have an effect on alertness. This proposal is based on the following assumptions. On the one hand, we can reasonably assume that the illuminance threshold required to have a significant effect on alertness during daytime will be at least as high as a threshold applicable to night-time exposure because we are more sensitive to lighting during the night. On the other hand, we can also reasonably assume that if a significant effect was found during daytime with a given illuminance, those effects will probably still be observed with an even higher illuminance. We should note that the Phipps-Nelson study was run with slightly sleep-deprived subjects and with a dim light background $(<5$ lux $)$, which could skew the results towards a higher sensitivity to light exposure than would have been observed with well-rested subjects or a bright background; but the purpose for now is more about defining a methodology than finding exact values. 
In both studies, fluorescent tubes were used as the light source: Philips Color $8404100 \mathrm{~K}$ fluorescent tubes in the (Cajochen) nighttime study and Thorn 2L (36 W) tubes in the (Phipps-Nelson) daytime one. From Figure 4 one can read that the 'circadian-equivalent' lower bound illuminance value of 300 lux for $4100 \mathrm{~K}$ fluorescent light (which gave a $100 \%$ alerting in the Cajochen et al study) would be 190 lux for the CIE illuminant D65 (overcast sky). Similarly, we find that a 'circadian-equivalent' lower bound illuminance of 210 is determined for CIE illuminant D55 (sunlight), and 180 lux for CIE illuminant D75 (clear blue sky light). The Phipps-Nelson study used a mean eye illuminance of 1056 lux as the bright light condition to evaluate daytime alerting effects. We do not have spectral data for the particular fluorescent tubes that were used but can assume that they approximate the Illuminant F7 (Daylight Fluorescent) reasonably well. We then have all the data necessary to determine the 'circadian-equivalent' upper bound illuminance of 1056 lux of fluorescent (F7) light for the CIE illuminant D65 ${ }^{44}$, which we find to be 870 lux. The same process leads to a 'circadian-equivalent' upper bound illuminance for the CIE illuminant D55 of 960 lux and for the CIE illuminant D75 of 830 lux. As one would expect, the 'bluer' spectrum (D75) corresponds to the lowest equivalent illuminance threshold in both cases as it provides more 'circadian-effective' light for a given number of visual lux.

Accounting for the noted uncertainties, a simple ramp-function can be proposed as a reasonable proxy to represent the likelihood that the vertical illuminance at a given point in time and for a given view direction is sufficient to affect the circadian system and have either circadian entrainment and/or subjective alertness effects: low likelihood ( $0 \%$ ) below the lower bound, and high likelihood (100\%) above upper bound, with a linear interpolation between these 
values. As with most biological effects, a response curve is more likely to be the reality than a linear ramp function. Though the Cajochen et $\mathrm{al}^{31}$ dose response itself was fitted with a much more complex, four-parameter logistic model, there is a steep linear component over the hundreds of lux range. The linear ramp can thus be considered as a first, simplistic step that has some basis in biology. It is likely to underestimate the non-visual response associated with a particular illuminance level (number of lux) though, and should be refined as more experimental data becomes available.

Given that 'circadian-equivalent' illuminance values and thresholds represent quantities that are - by definition - always relative to two source spectra whose respective potential to produce significant 'circadian' (non-visual) effects is compared, an absolute 'circadian-equivalent' illuminance value thus has no meaning by itself. When dealing with three light source spectra (overcast sky, clear sky and sunlight), it is thus preferable to choose a single and consistent daylight source of reference, say Illuminant D55, and gauge results against it. Before comparing partial (visual) illuminance values to threshold values, we convert the respective components due to overcast sky (D65) and clear blue sky (D75) into 'circadian-equivalent' illuminances for D55 sunlight. Then, all three can be added up and assessed against D55-relevant lower and upper thresholds, i.e. 210 lux and 960 lux thresholds respectively, as mentioned above.

The resulting ramp function expressed in terms of D55 equivalent is illustrated in Figure 5. As noted, these and other parameters will be refined in due course when advances in quantitative photobiology deliver more certain data for model calibration. 
Figure 5: Schematic showing ramp-function for likelihood of non-visual effect.

\subsection{Timing of exposure}

The timing of exposure determines the magnitude and direction of the circadian resetting effect for a given light exposure and therefore critical in determining the type of effect that can be expected, and whether it will be beneficial or detrimental for any given application. Given our incomplete knowledge about the interaction of light exposure timing, pattern, wavelength, intensity, duration and history, the boundaries are not yet defined, but nevertheless it is possible to delineate three distinct times of day from the photobiology findings discussed in section 2. These are illustrated in Figure 6 and can be summarized as follows:

- Early to mid-morning (6:00-10:00 h), where sufficient daylight illuminance can serve to phase advance the clock in the majority of people. (Note, about $75 \%$ of the population have an intrinsic circadian period $>24 \mathrm{~h}$ which requires a daily phase advance to entrain to the 24hour light-dark cycle ${ }^{45,46}$; a quarter however, require a delay shift to entrain which would be achieved by evening light exposure).

- Mid-morning to early evening (10:00 to 18:00 h), where high levels of daylight illuminance may lead to increased levels of subjective alertness without exerting substantial phase shifting effects on the clock.

- The rest as notional night-time (18:00 to 6:00 h), where daylight exposure that might trigger the non-visual effect is to be avoided so as not to disrupt the natural wake-sleep cycle (although see caveat above regarding individuals with a circadian period $<24 \mathrm{~h}$ ). 
We should note that the categorization of beneficial versus detrimental will depend on the type of activity/task that one has to do. For example, the threeepisode schedule described would be applicable to a hospital patient recovering from surgery. It would not necessarily be applicable to the night-shift nurses however, who have to work during the hours of darkness: their alertness will be increased with an exposure to bright light overnight (18:00 to 6:00 h), and they should limit light exposure during the day to facilitate sleep. Similarly, those individuals with a circadian period $<24 \mathrm{~h}$, who require a daily delay to entrain their circadian clocks, would benefit from obtaining evening light exposure and minimizing morning exposure.

Figure 6: The day is divided into three episodes according to the type of non-visual effect that applies to each one.

These three time episodes over the $24 \mathrm{~h}$-day can then be used to categorize timevaried light exposure over a day or a year according to their expected nonvisual effects by calculating the cumulative occurrence of non-visual effect likelihood within each category. This concept is further discussed in the following section and was applied for a range of climates, spaces and opening configurations in a residential environment for Part II of the study.

As noted, the timing factor should include not only the duration and time of occurrence but also the history of recent exposure. We do not know enough yet to warrant the additional complexity of including this factor, however, so we consider only time of occurrence in isolation of the duration and history of the exposure. 


\section{The 'circadian potential' of a space}

A daylit space will experience continuously changing natural illumination conditions over the day and from season to season. This sequence of conditions will be unique to every design and every building, site and location. With climate-based modeling methods, we can predict these variations from standardised weather data (as described in Part II) and can therefore devise ways to provide a more comprehensive understanding of how optimal a space might be in terms of its potential in providing 'circadian-effective' daylight over representative periods of a full year.

\subsection{Cumulative occurrence of non-visual effects}

The 'circadian potential' aims to provide the means to know where, when and to what extent circadian entrainment and alertness effects are likely to occur in a space. More specifically, for a given view direction at a given location in a space, it can be understood as how often over the year someone looking in that direction from that location would experience light levels that are above the required thresholds for inducing non-visual effects (such as entraining his/her circadian system or increasing his/her alertness). It therefore expresses the cumulative occurrence of experiencing non-visual effects over time, and can be compared to an absolute ' $100 \%$ potential' reference that would correspond to a situation where at any given moment over the year, the light exposure does 
surpass the required threshold. The comparison against a given threshold would here rely on the process described in sections 3.1 and 3.2.

An essential aspect of this potential relates to how the space is designed - and in particular to the position, size, orientation and type of openings it includes. Another essential aspect relates to how it is occupied: light will only be 'circadian-effective' if actually detected by an occupant - and occupants are known to move and look around in a space in numerous ways. Actual light exposure will therefore vary considerably depending on: (a) whether the occupant tends to look towards or away from available light sources; and, (b) the magnitude of the (diffuse) illumination in the space that results from one or more reflections of light once it has entered the space through an aperture (e.g. window or rooflight).

While the 'circadian potential' concept applies to a given view direction and head position, it could just as well be extended to an entire space: in this case, one could think of averaging individual 'circadian potentials' for a range of locations and view directions that would be representative of the full space, or of randomizing which location and view direction to pick at each moment (as proposed in Gochenour \& Andersen $2009^{47}$ for circadian potential evaluation in rowhouse apartments). One could even move to a further degree of subtlety by incorporating occupant behavior models and space use patterns, as also suggested but not attempted in 47 .

As the effect of light exposure can be very different depending on its timing over the day (beneficial versus detrimental depending on one's activity e.g.), the 'circadian potential' should not simply be the result of a cumulative 
exposure over time, but should be grouped according to the type of effect, i.e. within - in the simplified model proposed here - the three time episodes discussed in section 3.3. Ultimately, such a static model will have to be refined into a dynamic one, that offers enough flexibility to account for differences in duration, intensity, pattern and wavelength and better mimic the dynamic nature of circadian photoreception. The proposed model therefore represents an initial step in setting an appropriate framework to address this challenge.

In order to visualise the outcomes of such a model on an annual basis (whether based on dynamic hypotheses or, as is currently the case, static ones) and in a way that can actually be informative if incorporated into the design process, we developed a condensed visualisation format for 'circadian potential'. It combines the need to group time-varying data within three daily time episodes and to do this according to a finite set of representative view directions. This format is described in the next section.

\subsection{Graphical representation: the sombrero plot}

We devised a simple and intuitive graphical schema to express the 'circadian potential' of a given location and view direction as the cumulative non-visual effect occurring in each of the three times of the day identified independently.

We present the cumulative non-visual response occurring in the three defined times of day using a graphical device that we have called the 'sombrero' plot. Illustrated in Figure 7, the 'sombrero' plot shows the percentage of the cumulative occurrence of non-visual effects across the year for each of the three 
periods described in the previous section. The boundaries for the periods were thus set as follows:

- The effects of light on circadian resetting from 6:00 to 10:00 $\mathrm{h}$ are represented by the inner circle of the 'sombrero'

- The alerting effects of light from 10:00 to 18:00 $\mathrm{h}$ are represented by the middle circle of the 'sombrero'

- The light avoidance time from 18:00 to $6: 00 \mathrm{~h}$ is represented by the outer circle of the 'sombrero'.

The cumulative 'circadian potential' of light for these three times of day for a fixed location is apportioned to four quarter-segments in three concentric rings, shown in the lower panel in Figure 7:

- Each ring segment gives the cumulated percentage of that time period across the year for which the circadian potential (likelihood of having an effect) would be achieved for that view direction and at that location (i.e. multiple plots are used to characterize the variation in effect across a space - paper II).

- Each quarter circle corresponds to a different view direction (N, E, S and W e.g., or more generally 'bottom', 'top', 'left' and 'right') and is shaded according to a $0-100 \%$ false-colour scale ranging from light yellow $(100 \%$ likelihood of non-visual effect) to dark blue $(0 \%$ likelihood of effect).

In the example given, the 'sombrero' plot could be associated to a specific location in an interior space that would for instance include a South-West window (North is 'up'), for which we are interested to get an annual overview of light exposure for, say, a particular desk layout. The plot would tell us that, 
at that particular seating position, for someone looking mostly towards the South or West over the year from the late morning to the late afternoon (10:00 to 18:00 h), non-visual effects will likely be significant (close to $100 \%$ potential) compared to someone looking mostly to the North (80\%) or the East $(70 \%)$ during those hours of the day. If we are in a situation where morning light exposure (6:00 to 10:00 h) is critical in that space, we would observe that East or North view orientations might not be ideal (20\% to $40 \%)$ whereas if the priority is to avoid evening light (18:00 to 6:00 h), we would see that the space is likely to be suitable as is. This latter observation might seem obvious under latitudes below $60^{\circ}$ but is likely to become an influential design factor for locations closer to (or beyond) the arctic circles in the summer periods.

Given that the shaded value in the 'sombrero' plot is a cumulative measure, a value of, say, $40 \%$ could either represent a full 'circadian potential' (i.e. D55 circadian-equivalent light exposure above 960 lux in the proposed model) occuring for $40 \%$ of the time, or a $40 \%$ 'circadian potential' (D55 circadianequivalent light exposure above 210 lux but below 960 lux i.e. close to 510 lux with the chosen linear ramp-function) occurring for all of the time, or, as is more likely, something in between. The application of this concept in a modeling workflow and its ability to reveal critical space properties relevant to non-visual effects are discussed in Part II.

Figure 7: Visualization of 'circadian potential' as a sombrero plot, which expresses the cumulative occurrence of non-visual effects within each time period at a given location and for four viewing directions.

This representation actually offers a large degree of flexibility in what type of information can be displayed, depending on the designer's own priorities. If 
specific periods over the day or the year are more relevant than others (e.g. for a classroom space only used during limited daytime hours and the academic year), the 'circadian potential' could be adjusted to only evaluate light exposure within these periods. Or, if what the designer is looking for is to get a general idea of the space's potential to generate non-visual effects, metrics similar to the Acceptable Illuminance Extent (AIE) developed within the Lightsolve context $^{48}$ could be used, that look at what proportion of a space achieves a certain goal; this would condense the information into a single value per space (or per area of interest) for each view direction. To be more realistic, this synthesis could ultimately rely, as mentioned earlier, on estimated 'circadian lux' based on realistic human behaviour or activity patterns derived from a combination of established behavioural models in living/working spaces ${ }^{49,50}$ and of simultaneously computed glare models ${ }^{51,52}$ to ensure that the exposure patterns are realistic i.e. that they do not conflict with visual comfort requirements. This would constitute a challenging but interesting further enhancement of the proposed model.

\section{Conclusions}

A new model of non-visual effects which is an extension of that described by Pechacek, Andersen \& Lockley ${ }^{18}$ is presented in this paper. A key enhancement compared to the previous implementation resides in the introduction of the concept of a linear ramp-function from a lower to an upper vertical illuminance threshold that expresses the increasing potential for circadian effects. Another enhancement is the ability to treat light from the sun and sky independently, thereby accounting for the varying circadian efficiency of the daylight according to its spectral type, i.e. D55, D65 or D75. Additionally, in order to 
simplify data visualisation, we have introduced a novel graphical device to display the cumulative non-visual effects of light at a point in space as a function of view direction, in an intuitive and highly condensed way: the 'sombrero' plot provides a means of representing cumulative data which has properties of position (i.e. multiple plots can be used to show the distribution across a space) and view direction, in addition to incorporating changes due to the time of day. The simplicity of its graphical concept also allows a lot of flexibility in the type of data that are being condensed (over the year vs. periods of the year, over the space or areas of interest etc), so as to better respond to a designer's project priorities and to remain applicable as new photobiology findings will support the creation of inherently dynamic lighting models for non-visual effects.

The field of 'circadian daylighting' in architecture is a new one. The proposed approach aims to provide the basis to support a better understanding of the relative effect of certain design decisions on the overall 'circadian potential' of a space. As we have noted, the understanding of the dynamic properties of the circadian photoreception system continues to advance, and therefore we expect to refine and rerun our models as additional experimental data emerge. It is certainly too early at this stage to consider definitive guidelines for buildings in terms of predicted circadian potential. Notwithstanding this limitation, however, the basis is sufficiently robust to start formulating credible methodologies for circadian potential simulation and to start building an appropriate modeling framework for its investigation in the design context.

Ultimately, the complex inter-dependencies of intensity, duration, pattern, timing, light history and wavelength parameters affecting the circadian 
system's response during both day and night will have to be incorporated into a dynamic and flexible model. Recent photobiology studies have, for example, shown that non-visual photoreception is mediated by multiple photoreceptors depending on the intensity and duration of light exposure ${ }^{53}$. Under dim light conditions, or at the start of light exposure, the photopic visual system $V(\lambda)$ was shown to contribute at least equally to non-visual responses whereas at higher intensities, melanopsin-based blue-light sensitivity was shown to predominate for melatonin suppression. Lockley's group has also shown that the durationresponse function to light is non-linear with the greatest effect at the start of the light exposure ${ }^{54}$ and there seem to be time-of-day differences in the spectral circadian response $\mathrm{C}(\lambda)$, which are currently under investigation. Therefore, we will need to generate an inter-dependent function that links exposure duration with intensity and time of day. While we cannot determine an actual expression for it yet, we already know what the main variables are and the simplified and static model proposed here is a first step towards establishing its foundations.

Once more refined simulation models emerge that account for these interdependencies, we will then be able to test them against, say, patient data recorded in controlled laboratory studies or - ideally - in actual built environments (e.g. healthcare), to ensure that the models are applicable in real world design applications. New opportunities will then open up for how we might formulate future building guidelines that account for what are increasingly believed to be significant health, well-being and productivity effects related to the exposure of daylight.

\section{Acknowledgements}


The authors would like to acknowledge the support of the Velux Corporation who commissionned this study, and in particular the contributions and input of Nicolas Roy and Jens Christoffersen. Marilyne Andersen was also supported by the Ecole Polytechnique Fédérale de Lausanne and John Mardaljevic by De Montfort University. The authors also thank Christopher Pechacek, whose MSc thesis $^{55}$ - co-supervised by two of the authors (Andersen and Lockley) - served as a starting point for the proposed method and as the source for figures 2 and 4. 


\section{References}

1. Mardaljevic, J. (2000). Simulation of annual daylighting profiles for internal illuminance. Lighting Research and Technology, 32(3):111-118.

2. Reinhart C.F., Herkel, S. (2000). The simulation of annual daylight illuminance distributions - a state-of-the-art comparison of six RADIANCE-based methods. Energy and Buildings, 32(2):167-187.

3. Mardaljevic, J., Heschong, L., Lee, E. (2009). Daylight metrics and energy savings. Lighting Research and Technology, 41(3):261-283.

4. Webb, A. R. (2006). Considerations for lighting in the built environment: Non-visual effects of light. Energy and Buildings, 38(7):721-727.

5. Collins, B. L. (1976). Review of the psychological reaction to windows. Lighting Research and Technology, 8(2):80-88.

6. Heschong, L. (2002). Daylighting and human performance. ASHRAE Journal, 44(6):65-67.

7. Lockley, SW. and Dijk, D. J. (2002). Functional genomics of sleep and circadian rhythm. Journal of Applied Physiology, 92:852-862.

8. Wehr, T., Aeschbach, D., and Duncan, W. J. (2001). Evidence for a biological dawn and dusk in the human circadian timing system. The Journal of Physiology, 535(3):937-951.

9. Lockley, SW. (2009). Influence of light on circadian rhythmicity in humans. L. R. Squire (Ed.) Encyclopaedia of Neuroscience. Oxford, UK.

10. Veitch, J., van den Beld, G., Brainard, G., and Roberts, J. (2004). Ocular lighting effects on human physiology and behaviour. CIE Publication, Vienna, Austria, 158.

11. Boyce, P. R., Beckstead, J. W., Eklund, N. H., Strobel, R. W., and Rea, M. S. (1997). Lighting the graveyard shift: The influence of a daylightsimulating skylight on the task performance and mood of night-shift workerst. Lighting Research and Technology, 29(3):105-134.

12. Wilson, L. (1972). Intensive care delirium. Archives of Internal Medicine, 130:225-226.

13. Walsh, J., Rabin, B., Day, R., Williams, J., Choi, K., and Kang, J. (2005). The effect of sunlight on postoperative analgesic medication use: A prospective study of patients undergoing spinal surgery. Psychosomatic Medicine, 67(1):156-163. 
14. Beauchemin, K. and Hays, P. (1998). Dying in the dark: Sunshine, gender and outcomes in myocardial infarction. The Royal Society of Medicine, 91:352-354.

15. Riemersma-van der Lek, R., Swaab, D., Twisk, J., Hol, E., Hoogendijk, W., and Van Someren, E. (2008). Effect of bright light and melatonin on cognitive and noncognitive function in elderly residents of group care facilities: a randomized controlled trial. JAMA-The Journal of the American Medical Association, 299(22):2642-55.

16. Mills, P., Tomkins, S., and Schlangen, L. (2007). The effect of high correlated colour temperature office lighting on employee wellbeing and work performance. Journal of Circadian Rhythms, 5(1):2.

17. Viola, A., James, L., Schlangen, L., and Dijk, D. (2008). Blue-enriched white light in the workplace improves self-reported alertness, performance and sleep quality. Scandinavian Journal of Work Environment and Health, 34(4):297-306.

18. Pechacek, C. S., Andersen, M., and Lockley, S. W. (2008). Preliminary method for prospective analysis of the circadian efficacy of (day) light with applications to healthcare architecture. Leukos, 5(1):1-26.

19. Rea, M, Figueiro, M, Bierman, A, Bullough, J. (2010). Circadian light, Journal of Circadian Rhythms 8 (1)

20. Figueiro, MG, Brons, JA, Plitnick, B, Donlan, B, Leslie, RP, Rea, MS. (2011). Measuring circadian light and its impact on adolescents, Lighting Research and Technology 43(2)

21. Leslie, RP, Radetsky, LC, Smith, AM. (in press). Conceptual design metrics for daylighting, Lighting Research and Technology

22. Linhart, F, Scartezzini, J-L, Münch, M. (2009). Daylight exposure and circadian efficiency in office rooms equipped with anidolic daylighting systems, Proceedings of CISBAT 2009

23. Arsenault, H, Hébert, M, Dubois, M-C. (2011). Effects of glazing colour types on daylight quality, arousal and switch-on patterns for electric lights in a scaled office room, Glass Performance Days Conference 2011

24. Gall, D, Bieske, K. (2004). Definition and measurement of circadian radiometric quantities, In Proceedings of the 2004 CIE symposium 2004

25. Rea, MS, Figueiro, MG, Bullough, JD, Bierman, A. (2005). A model of phototransduction by the human circadian system, Brain Research Review $50(2)$ 
26. Al Enezi, J, Revell, V, Brown, T, Wynne, J, Schlangen, L, Lucas, R. (2011). A "Melanopic" Spectral Efficiency Function Predicts the Sensitivity of Melanopsin Photoreceptors to Polychromatic Lights, Journal of Biological Rhythms 26(4)

27. Bellia, L, Bisegna, F, Spada, G. (2011). Lighting in indoor environments: Visual and non-visual effects of light sources with different spectral power distributions, Building and Environment 46 (10)

28. Brainard, G. C., Hanifin, J. P., Greeson, J. M., Byrne, B., Glickman, G., Gerner, E., and Rollag, M. D. (2001). Action spectrum for melatonin regulation in humans: Evidence for a novel circadian photoreceptor. Journal of Neuroscience, 21(16):6405-6412.

29. Thapan K, Arendt J, Skene DJ. (2001). An action spectrum for melatonin suppression: evidence for a novel non-rod, non-cone photoreceptor system in humans. Journal of Physiology 535 (Pt 1):261-7.

30. Berson DM, Dunn FA, Takao M. (2002). Phototransduction by retinal ganglion cells that set the circadian clock. Science. 295(5557):1070-3.

31. Cajochen, C., Zeitzer, J., Czeisler, C., and Dijk, D. (2000). Dose-response relationship for light intensity and ocular and electroencephalographic correlates of human alertness. Behavioral Brain Research, 115:75-83.

32. Rüger M., Gordijn, M.C.M., Beersma, D.G.M., de Vries, B., Daan, S. (2006). Time-of-day-dependent effects of bright light exposure on human psychophysiology: comparison of daytime and nighttime exposure. American Journal of Physiology - Regulatory, Integrative and Comparative Physiology 290: R1413-R1420.

33. Phipps-Nelson, J., Redman, J., Dijk, D., and Rajaratnam, S. (2003). Daytime exposure to bright light, as compared to dim light, decreases sleepiness and improves psychomotor vigilance performance. Sleep, 26(6):695-700.

34. Badia, P., Myers, B., Boecker, M., Culpepper, J., and Harsh, J. (1991). Bright light effects on body temperature, alertness, eeg and behavior. Physiology \& Behavior, 50:583-8.

35. Lafrance, C., Dumont, M., Lesperance, P., and Lambert, C. (1998). Daytime vigilance after morning bright light exposure in volunteers subjected to sleep restriction. Physiology \& Behavior, 63:803-10. 
36. Khalsa SB, Jewett ME, Cajochen C, Czeisler CA. (2003) A phase response curve to single bright light pulses in human subjects. Journal of Physiology 549(3): 945-952.

37. Sletten T.L., Revell V.L., Middleton B., Lederle K.A., Skene D.J. (2009). Age-related changes in acute and phase-advancing responses to monochromatic light. J Biol Rhythms 24(1):73-84.

38. Münch M., Kobialka S., Steiner R., Oelhafen P., Wirz-Justice A., Cajochen C. (2006). Wavelength-dependent effects of evening light exposure on sleep architecture and sleep EEG power density in men. Am J Physiol Regul Integr Comp Physiol. 290(5):R1421-8.

39. Gronfier C, Wright KP Jr, Kronauer RE, Jewett ME, Czeisler CA. Am J Physiol Endocrinol Metab. (2004). Efficacy of a single sequence of intermittent bright light pulses for delaying circadian phase in humans. 287(1):E174-81

40. Smith KA, Schoen MW, Czeisler CA. (2004). Adaptation of human pineal melatonin suppression by recent photic history. Journal of Clinical Endocrinology \& Metabolism 89(7):3610-4.

41. Hébert M, Martin SK, Lee C, Eastman CI. (2002). The effects of prior light history on the suppression of melatonin by light in humans. Journal of Pineal Research 33(4):198-203.

42. Lockley SW, Evans EE, Scheer FA, Brainard GC, Czeisler CA, Aeschbach D. (2006). Short-wavelength sensitivity for the direct effects of light on alertness, vigilance, and the waking electroencephalogram in humans. Sleep. 29(2):161-8.

43. Mardaljevic, J. (2008). Sky model blends for predicting internal illuminance: a comparison founded on the BRE-IDMP dataset. Journal of Building Performance Simulation 1(3):163-173.

44. Commission Internationale de l'Eclairage (2006). Colorimetry - Part 2: CIE Standard Illuminants.

45. Czeisler CA, Duffy JF, Shanahan TL, Brown EN, Mitchell JF, Rimmer DW, Ronda JM, Silva EJ, Allan JS, Emens JS, Dijk DJ, Kronauer RE. (1999). Stability, precision, and near-24-hour period of the human circadian pacemaker. Science. 284(5423):2177-81.

46. Duffy JF, Cain SW, Chang AM, Phillips AJ, Münch MY, Gronfier C, Wyatt JK, Dijk DJ, Wright KP Jr, Czeisler CA. (2011). Sex difference in the near-24-hour intrinsic period of the human circadian timing system. 
Proceedings of the National Academy of Science, USA. 108 Suppl 3:15602-8.

47. Gochenour S., Andersen M. (2009). Circadian Effects of Daylighting in a Residential Environment, In Proceedings of Lux Europa 2009 - 11th European Lighting Conference, Istanbul, September 9-11, 2009.

48. Kleindienst, S., Andersen, M. (2012). Comprehensive Annual Daylight Design through a Goal-Based Approach, Building Research and Information, in press

49. Intille, SS, Larson, K, Beaudin, JS, Nawyn, J, Tapia, EM, Kaushik, P. (2005). A living laboratory for the design and evaluation of ubiquitous computing technologies, In Conference on Human Factors in Computing Systems; Portland, Oregon

50. Lühr, S, West, G, Venkatesh, S. (2007). Recognition of emergent human behaviour in a smart home: A data mining approach, Pervasive and Mobile Computing 3(2)

51. Wienold, J, Christoffersen, J, Evaluation methods and development of a new glare prediction model for daylight environments with the use of CCD cameras, Energy and Buildings 38 (7), 2006

52. J Wienold, Dynamic daylight glare evaluation, In Proc. of International IBPSA Conference, 2009

53. Gooley, JJ, Rajaratnam, SMW, Brainard, GC, Kronauer, RE, Czeisler, CA, Lockley, SW. (2010). Spectral responses of the human circadian system depend on the irradiance and duration of exposure to light, Science Translational Medicine 2(31)

54. Gronfier, C, Wright KP, Kronauer, RE, Jewett, ME, Czeisler, CA. (2004). Efficacy of a single sequence of intermittent bright light pulses for delaying circadian phase in humans, Am J Physiol Endocrinol Metab 287(1)

55. Pechacek, C. (2008). Space, light, and time: Prospective analysis of circadian illumination for health-based daylighting with applications to healthcare architecture. M.Sc. in Architectural Studies (SMArchS), Massachusetts Institute of Technology, Cambridge MA, USA. 


\section{LIST OF FIGURES}

Figure 1: Spectral responses of visual system (photopic curve $V(\lambda)$ ) and circadian system (melanopsin action spectrum $C(\lambda)$ ).

Figure 2: Uncertainties related to context variability and space properties integrated in a relationship map between measurable (scientific) parameters and design elements.

Figure 3: Spectral power distribution for CIE daylight illuminants associated to the three daylight sources alongside normalized photopic and circadian sensitivity curves $\mathrm{V}(\lambda)$ and $\mathrm{C}(\lambda)$.

Figure 4: Illuminance necessary to achieve a desired relative alerting effect for a selection of well-known illuminants.

Figure 5: Schematic showing ramp-function for likelihood of non-visual effect.

Figure 6: The day is divided into three episodes according to the type of nonvisual effect that applies to each one.

Figure 7: Visualization of 'circadian potential' cumulative occurrence as a sombrero plot, which expresses the cumulative occurrence of non-visual effects within each time period at a given location and for four viewing directions. 


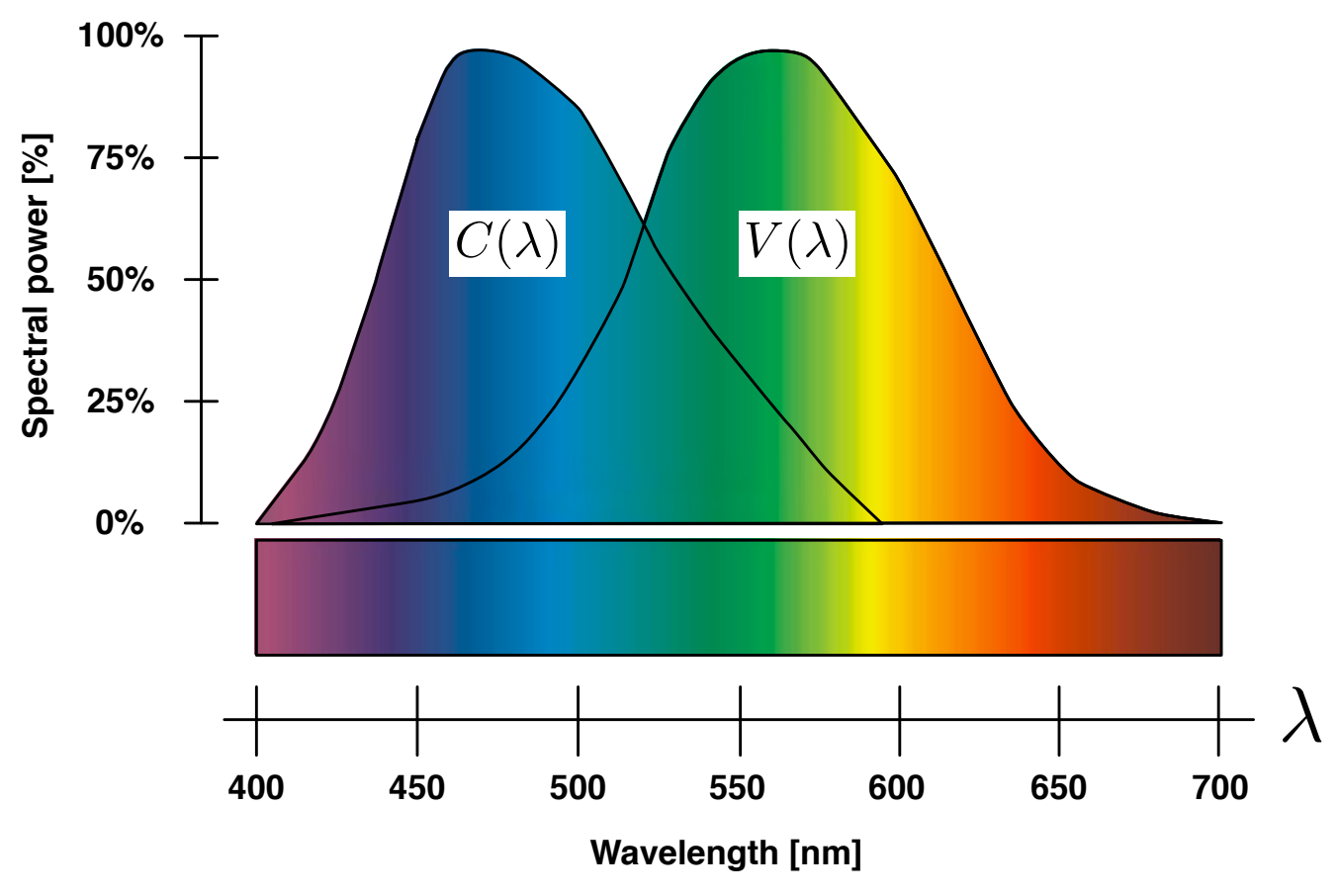

Figure 1: Spectral responses of visual system (photopic curve $V(\lambda)$ ) and circadian system (melanopsin action spectrum $C(\lambda)$ ). 


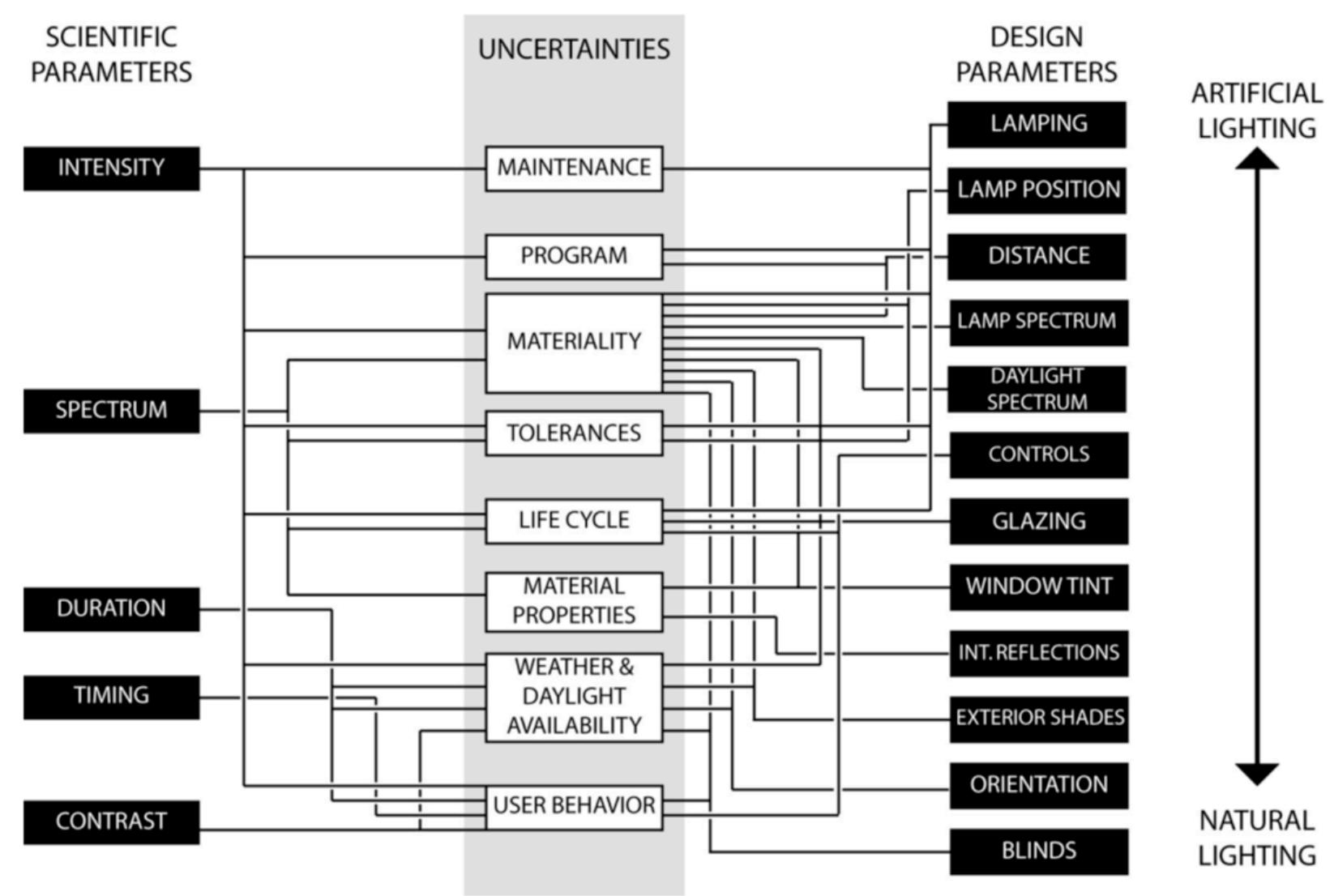

Figure 2: Uncertainties related to context variability and space properties integrated in a relationship map between measurable (scientific) parameters and design elements. 

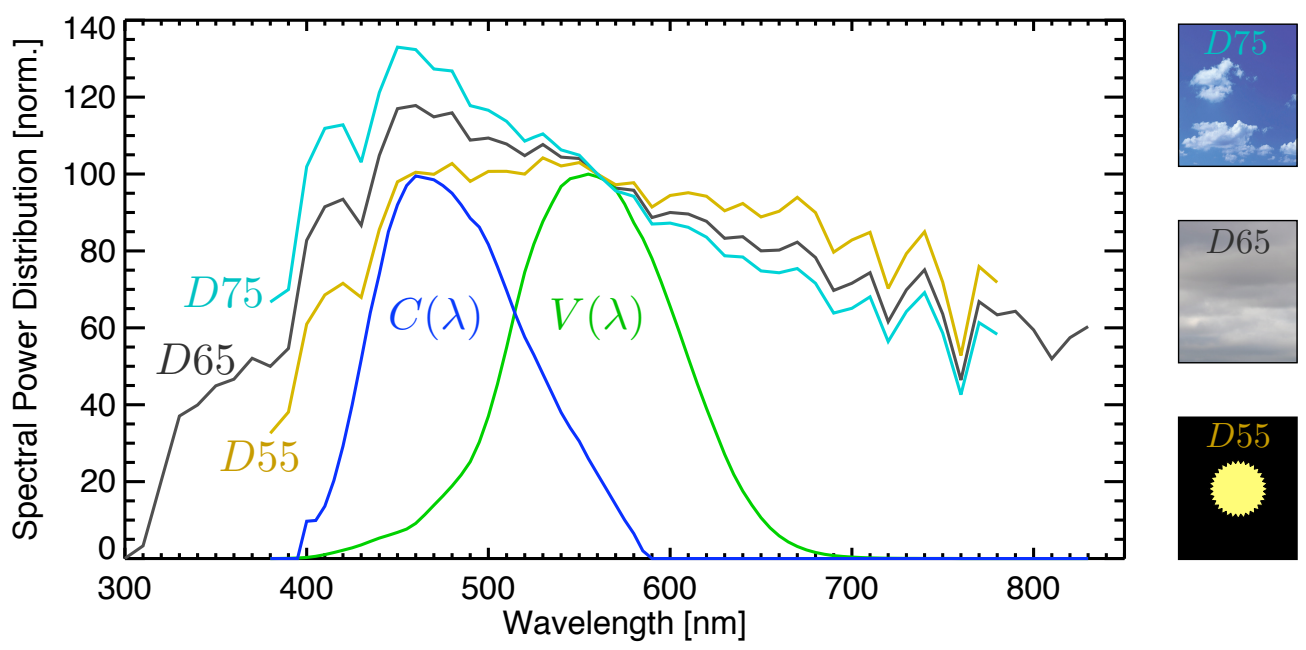

Figure 3: Spectral power distribution for CIE daylight illuminants associated to the three daylight sources alongside normalized photopic and circadian sensitivity curves $\mathrm{V}(\lambda)$ and $\mathrm{C}(\lambda)$. 


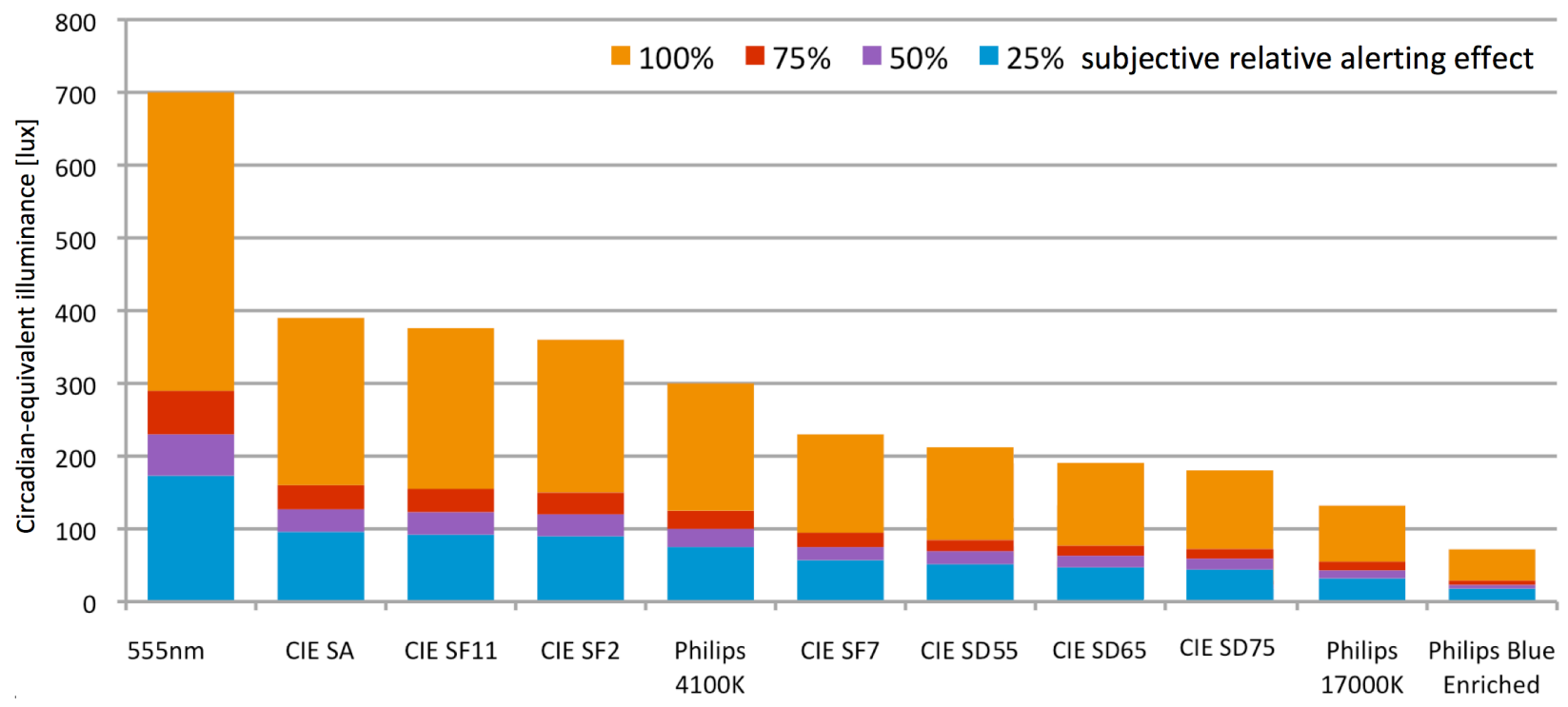

Figure 4: Illuminance necessary to achieve a desired relative alerting effect for a selection of well-known illuminants . 


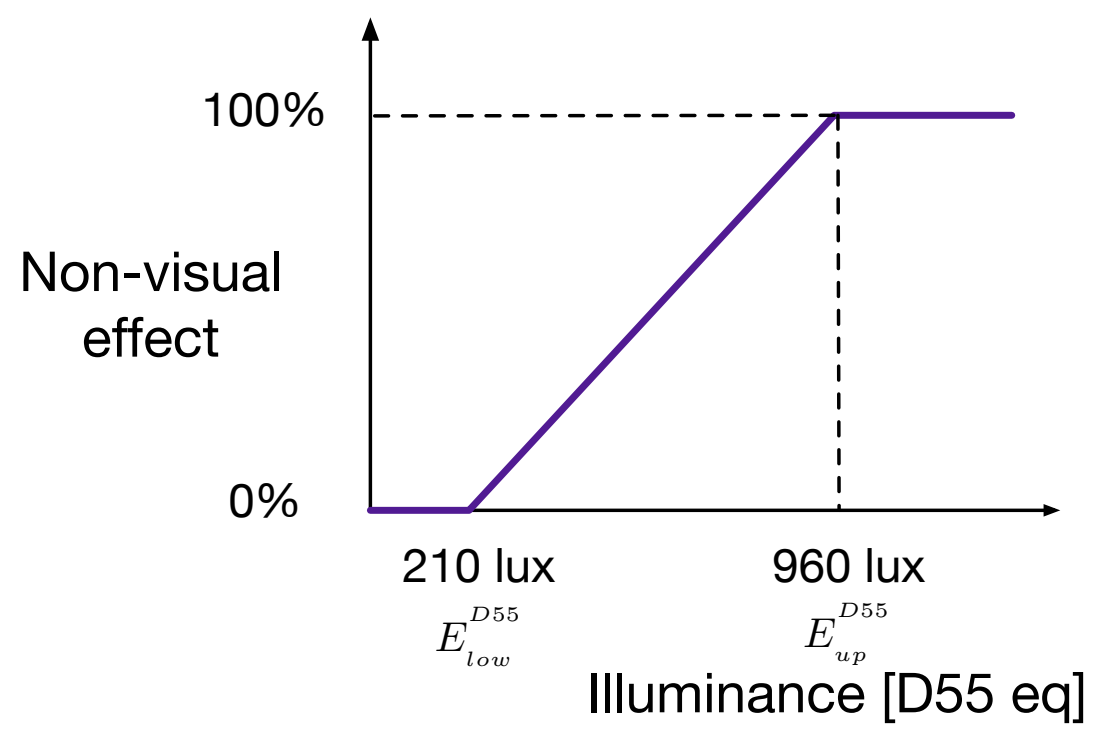

Figure 5: Schematic showing ramp-function for likelihood of non-visual effect. 


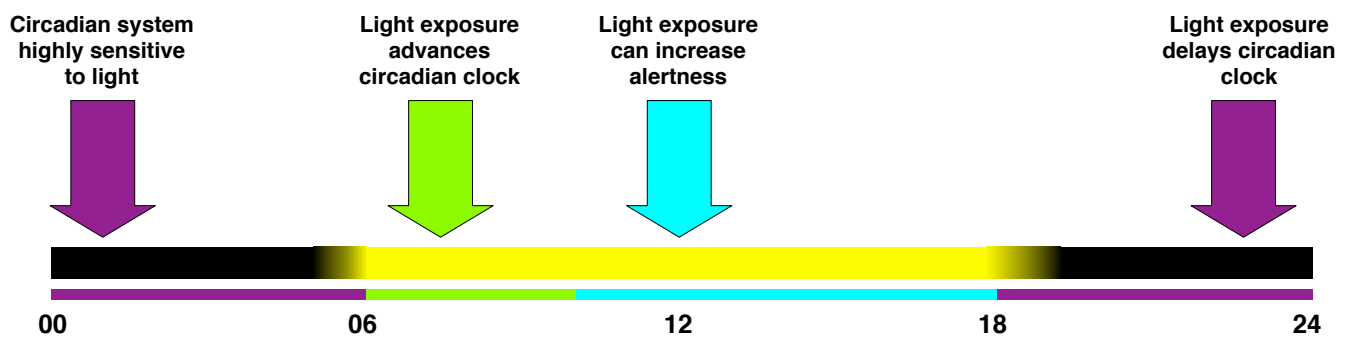

Figure 6: The day is divided into three episodes according to the type of nonvisual effect that applies to each one. 

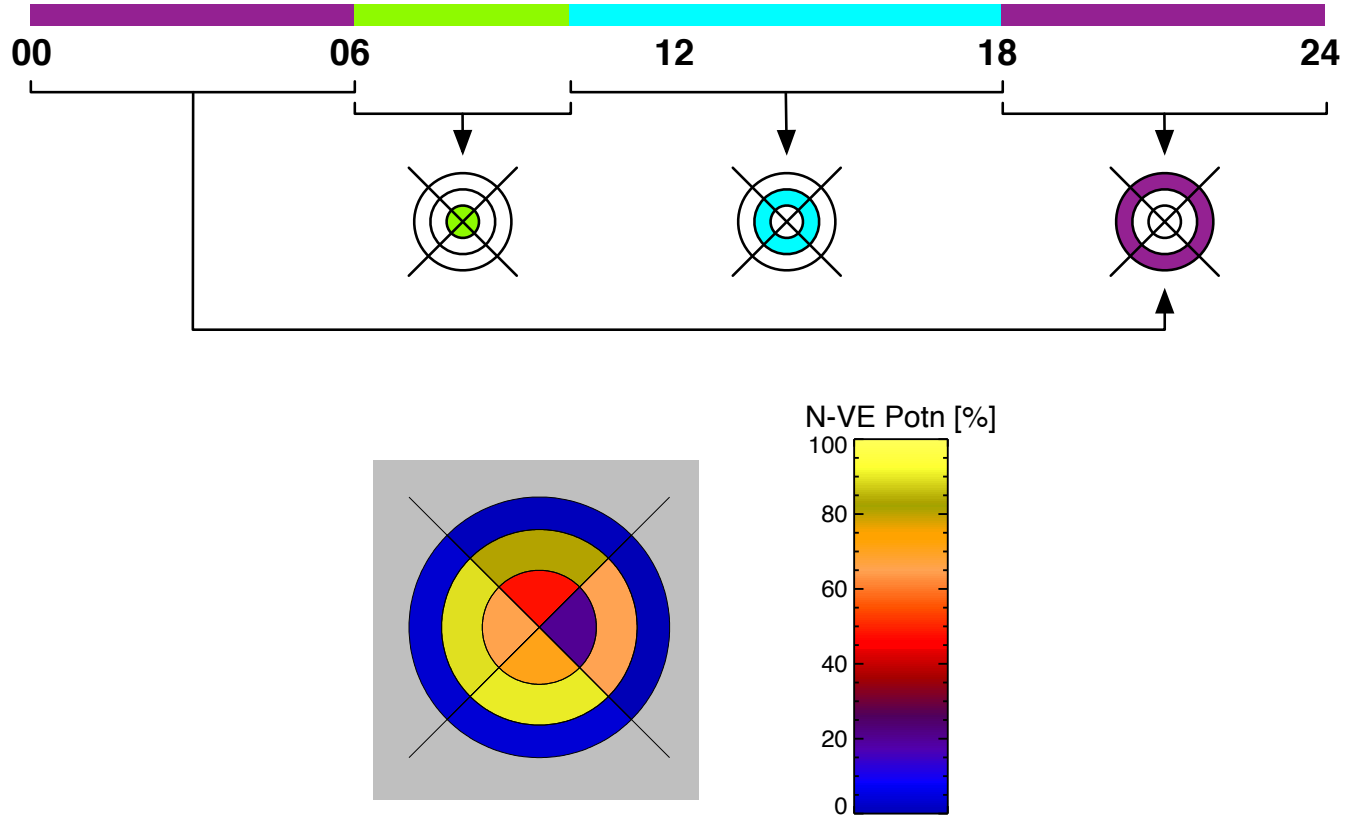

Figure 7: Visualization of 'circadian potential' cumulative occurrence as a sombrero plot, which expresses the cumulative occurrence of non-visual effects within each time period at a given location and for four viewing directions. 